\title{
La naturaleza de los aplicativos de movilidad
}

\author{
Mayra Alejandra de la Rosa Carrillo \\ Abogada por la Universidad de Lima. \\ Máster en Derecho (LLM) y Maestría en Ciencias (MSc) por la \\ Queen Mary University of London. \\ Miembro Asociado de ADV Editores - Revista ADVOCATUS.
}

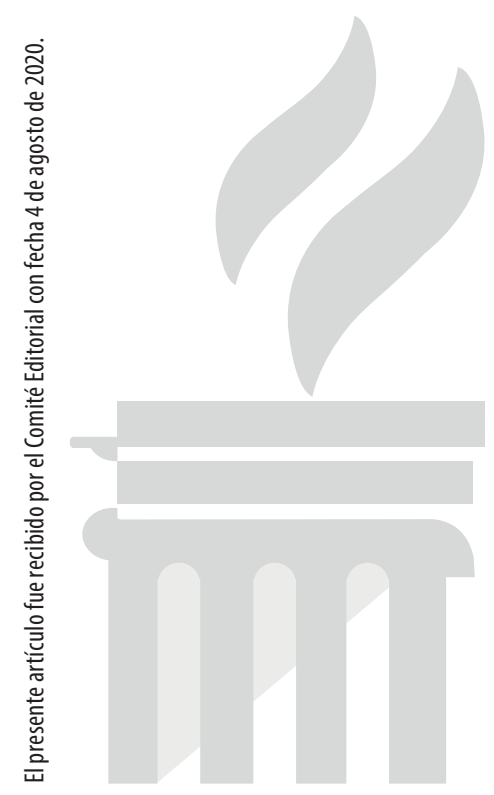

SUMARIO:

I. Introducción.

II. Pronunciamientos de INDECOPI respecto a la naturaleza de los aplicativos de movilidad.

1. Oficio $\mathrm{N}^{\circ} 888-2013-\mathrm{MML} / \mathrm{GTU}$.

2. Ordenanza $\mathrm{N}^{\circ} 1684-\mathrm{MML}$.

3. La revisión de la publicidad difundida por las empresas operantes en el mercado.

III. De las relaciones jurídicas existentes.

1. Relación jurídica 1: Aplicativo - Usuario Conductor.

2. Relación jurídica 2: Aplicativo - Usuario Pasajero.

3. Relación jurídica 3: Usuario Conductor - Usuario Pasajero.

IV. Conclusiones. 


\title{
RESUMEN:
}

Los aplicativos de movilidad son un fenómeno reciente en el Perú que ha causado una revolución en la concepción de las relaciones jurídicas entre consumidor y proveedor. La autora analiza las resoluciones de la autoridad competente respecto de la naturaleza de estos aplicativos de forma que se pueda descubrir su posicionamiento dentro del ordenamiento jurídico peruano.

Palabras clave: aplicativos de movilidad, taxi, derecho del consumidor, INDECOPI, transporte.

\begin{abstract}
:
Ride-hailing apps are a recent phenomenon in Peru that have caused a revolution in the legal conception of the relationship between consumer and provider. The author analyzes the competent authority's rulings regarding their nature, so that their positioning inside the legal system can be discovered.

Keywords: ride-hailing apps, taxi, consumer protection, INDECOPI, transportation.
\end{abstract}

\section{INTRODUCCIÓN}

Desde hace más de 5 años, los peruanos hemos experimentado una nueva forma de satisfacer nuestras necesidades de movilidad haciendo solo un clic desde nuestros celulares o smartphones. Nos encontramos conviviendo diariamente con plataformas cuya finalidad es poder optimizar nuestro tiempo, promover la interconectividad y mejorar la experiencia del usuario, versus el servicio tradicional. La interrogante que surge entonces es: ¿cómo han sido categorizados los aplicativos de movilidad dentro del actual sistema jurídico?

\section{PRONUNCIAMIENTOS DE INDECOPI RES- PECTO A LA NATURALEZA DE LOS APLI- CATIVOS DE MOVILIDAD}

Con la llegada de los aplicativos de movilidad, se inició un nuevo modelo de negocio y a su vez una nueva forma de satisfacer la demanda de movilidad de los peruanos. Esta nueva dinámica económica no fue ajena ni a los consumidores, quienes cada vez demandaban más estos servicios, ni al organismo encargado de velar por los derechos de los consumidores.

En ese sentido, la Secretaría Técnica de la Comisión de Competencia Desleal del Instituto Nacional de Defensa de la Competencia y de la Protección de la Propiedad Intelectual —INDECOPI; en adelante, la "Secretaría"—, determinó desde el año 2013 abrir varios procedimientos administrativos sancionadores contra algunas de las empresas de aplicativos de movilidad operantes en el mercado peruano en ese momento' por presunta comisión de actos de competencia desleal en la modalidad de engaño y violación de normas ${ }^{2}$.

1. Cfr. Expediente de Supervisión N 051-2014/GSF correspondiente a la empresa Easy Taxi Perú S.A.C; Expediente de Supervisión N 072-2014/GSF correspondiente a la empresa Maxi Mobility Perú S.A.C.; Expediente de Supervisión Nº73-2014/GSF correspondiente a la empresa SPE S.A.C.; Expediente de Supervisión Nº51-2014/GSF correspondiente a la empresa AutoTaxi Satelital S.A.C.

2. Los artículos 8 y 14 del Decreto Legislativo 1044, Ley de Represión de la Competencia Desleal disponen lo siguiente:

Artículo 8.- Actos de engaño.-

8.1.- Consisten en la realización de actos que tengan como efecto, real o potencial, inducir a error a otros agentes en el mercado sobre la naturaleza, modo de fabricación o distribución, características, aptitud para el uso, calidad, cantidad, precio, condiciones de venta o adquisición y, en general, sobre los atributos, beneficios o condiciones que corresponden a los bienes, servicios, establecimientos o transacciones que el agente económico que desarrolla tales actos 
Lo anterior con la finalidad de determinar su naturaleza; por cuanto, para criterio de la Secretaría, estas empresas estaban inobservando los permisos habilitantes para el desarrollo de la actividad de "taxi"; y a la par brindaban información engañosa a los consumidores, pues públicamente se identificaban como "empresas de taxi" cuando realmente operan como plataformas digitales.

La Secretaría sustentó la apertura del inicio del procedimiento sancionador de oficio contra estas empresas a través de los siguientes argumentos:

\section{Oficio $\mathrm{N}^{\circ} 888-2013-\mathrm{MML} / \mathrm{GTU}$.}

El Oficio $N^{\circ}$ 888-2013-MML/GTU —en adelante, el "Oficio" - proveniente de la Gerencia de Transporte Urbano de la Municipalidad Metropolitana de Lima, que principalmente menciona lo siguiente:

“(...) la Ordenanza No. 1684-MML prescribe que la autorización de servicio es el título habilitante que autoriza a una persona natural o jurídica para la prestación del servicio de taxi en Lima Metropolitana bajo la modalidad de Taxi Independiente, Taxi Estación o Taxi Remisse. Asimismo, dicha norma también dispone que todo vehículo del servi- cio de taxi deberá contar con su respectiva Tarjeta Única de Circulación emitido por la Gerencia de Transporte Urbano de la Municipalidad Metropolitana de Lima (GTU)." —El subrayado es nuestro-.

\section{Ordenanza N ${ }^{\circ}$ 1684-MML.}

La Ordenanza N 1684-MML, Ordenanza que regula la prestación del Servicio de Taxi en Lima Metropolitana —en adelante, la "Ordenanza"-, que principalmente indica lo siguiente:

"25. Servicio de Taxi: Es el servicio de transporte público especial de personas de ámbito provincial prestado en vehículos de la categoría vehicular 11 conforme con la clasificación establecida en el RNV, que tiene por objeto la movilización de personas desde un punto de origen hasta uno de destino señalado por quien lo contrata, de forma individual y por la capacidad total del vehículo. El servicio de taxi se caracteriza por su no sujeción a rutas, itinerarios y horarios. (...)". - El subrayado es nuestro-.

Al respecto es importante señalar que en el año 2013, la Municipalidad Metropolitana de Lima a través del Oficio y la Ordenanza estaba efectivamente facultada para solicitar ambas autorizaciones: (a) la autorización habilitante y

pone a disposición en el mercado; o inducir a error sobre los atributos que posee dicho agente, incluido todo aquello que representa su actividad empresarial.

(...)

8.3.- La carga de acreditar la veracidad y exactitud de las afirmaciones objetivas sobre los bienes o servicios corresponde a quien las haya comunicado en su calidad de anunciante.

8.4.- En particular, para la difusión de cualquier mensaje referido a características comprobables de un bien o servicio anunciado, el anunciante debe contar previamente con las pruebas que sustenten la veracidad de dicho mensaje.

Artículo 14.- Actos de violación de normas.-

14.1.- Consisten en la realización de actos que tengan como efecto, real o potencial, valerse en el mercado de una ventaja significativa derivada de la concurrencia en el mercado mediante la infracción de normas imperativas. A fin de determinar la existencia de una ventaja significativa se evaluará la mejor posición competitiva obtenida mediante la infracción de normas.

14.2.- La infracción de normas imperativas quedará acreditada:

(...)

b) Cuando la persona concurrente obligada a contar con autorizaciones, contratos o títulos que se requieren obligatoriamente para desarrollar determinada actividad empresarial, no acredite documentalmente su tenencia. En caso sea necesario, la autoridad requerirá a la autoridad competente un informe con el fin de evaluar la existencia o no de la autorización correspondiente. 
(b) la tarjeta única de circulación. No obstante lo anterior, esta facultad se encontraba estrictamente vinculada a empresas cuyo giro de negocio o actividad principal sea la prestación de servicio de transporte privado de pasajeros en la modalidad de "taxi". Partiendo de esta premisa, resulta conveniente señalar que los servicios brindados a través de los aplicativos de movilidad no se vinculan en absoluto a servicios de taxi en ninguna de sus categorías -independiente, estación o remisse-.

De hecho, este fue uno de los principales argumentos de defensa de más de una de las empresas de aplicativo de movilidad aduciendo que:

\section{"(...) de conformidad con la Ordenanza que} regula la prestación del servicio de taxi en Lima Metropolitana, el contrato de transporte de taxi vincularía a los usuarios del servicio con los prestadores de dicho servicio y no se establecería que la empresa que intermedia la demanda y la oferta del servicio de taxi y que permite interconectar a usuarios y taxistas prestadores del servicio, forme parte del contrato de transporte de taxi y/o que dicha empresa intermediadora se encontraría obligada a obtener algún tipo de habilitación especial por parte de la Municipalidad de Lima y/o que las autorizaciones de los vehículos (las Tarjetas Únicas de Circulación) debieran ser emitidas a nombre de la empresa intermediador (....)."3

-El subrayado es nuestro-.

Los aplicativos de movilidad no son más que plataformas diseñadas con el propósito que los usuarios puedan acceder a servicios en forma automatizada y optimizando tiempo. Dicho esto, a través de la tecnología, permiten fungir de intermediarios del sistema de transporte privado de pasajeros de forma tal que un usuario pasajero pueda conocer en forma anticipada el precio, la ruta, las características del vehículo y los datos de quien lo recogerá para movilizarse de un punto $A$ hacia un punto $B$.

Finalmente, parte de los argumentos de las empresas de aplicativos de movilidad fiscalizadas fue evidenciar la redacción del objeto social incluido en sus estatutos, los cuales no se encontraban relacionados a una empresa de transporte pues; como reiteramos, los aplicativos de movilidad usan la tecnología para interconectar demanda y oferta de transporte, mas no se crearon con el propósito de ofrecer servicios de transporte per se, sea en forma pública o privada.

\section{La revisión de la publicidad difundida por las empresas operantes en el mercado.}

En segundo lugar, respecto al argumento planteado por la Secretaría señalando que la publicidad podría llegar a suponer que los servicios corresponden a "taxi", debemos señalar que esto no sería correcto por cuanto, como bien lo ha indicado INDECOPI en sus lineamientos sobre competencia desleal y publicidad comercial, la publicidad deberá interpretarse en forma integral y superficial, no considerando interpretaciones alambicadas o que no formen parte de un análisis general de un consumidor razonable. ${ }^{4}$

Por lo tanto, si las empresas de aplicativos de movilidad difunden sus servicios utilizando palabras tales como "conectan", "intermedian", "fungen", "facilitan", "median", "canalizan", entre otros términos, la solicitud de servicios de transporte entre pasajeros y conductores, ipor qué habría que vincularla a una actividad que ciertamente no realizan? ¿Por qué esta conducta podría ser categorizada como un acto de engaño?

La finalidad del artículo 8 -actos de engaño- de la Ley de Represión de la Competencia Desleal es proteger a los consumidores de

3. Resolución N 100-2015-CD1-INDECOPI, 12.

4. Instituto Nacional de Defensa de la Competencia y de la Protección de la Propiedad Intelectual, Lineamientos sobre competencia desleal y publicidad comercial (Lima: INDECOPI, 2018), 12. 
la asimetría informativa en que se encuentran dentro del mercado con relación a los proveedores de bienes y servicios, quienes debido a su experiencia en el mercado han adquirido y utilizan, de mejor manera, la información relevante sobre las características de los servicios y productos que ofrecen.

Ahora bien, la razón tras del uso de las palabras antes descritas por parte de las empresas de aplicativos de movilidad es justamente disociar el supuesto vínculo como empresa de transporte; pues, nuevamente, estamos frente a un modelo de negocio disruptivo ${ }^{5}$ el cual no obliga a las empresas a contar con una flota de autos, conductores en planilla o rutas asignadas de transporte precisamente por eso, porque no fueron constituidas como empresas de transporte y claramente no es ese mensaje el que desean brindar las empresas dedicadas a esta nueva industria.

En los casos materia de análisis, la Secretaría ha cuestionado inclusive la forma de comunicar la prestación del servicio, como si fuera inviable dar a conocer las ventajas o beneficios del servicio mediante el aplicativo versus el servicio de transporte tradicional sin trastocar nuevamente su cuestionamiento como "empresa de taxi".

Pues bien, es importante resaltar lo siguiente: la forma como se realiza la comunicación de un servicio o sus ventajas, siempre que esta información sea objetiva, clara, verídica y no induzca a error respecto a lo que se ofrece, no debería ser debatida, sino mas bien entendida - y de ser necesario, previa colaboración de la industria a la cual se está fiscalizando-.

"Pedir un taxi nunca ha sido tan fácil. Con nuestra App para IOS y Android puedes llamar a un taxi desde cualquier lugar de la ciudad y ver el recorrido mientras nuestro taxi llega a tu ubicación." "Conectamos al taxista directamente con el pasajero!" "NO SOMOS UNA CENTRAL, somos un sistema que conecta al pasajero de manera directa con el taxista. Esto resulta ser un servicio más rápido para el pasajero y para que todos los taxistas cerca del pasajero puedan atender un servicio. (...)." ${ }^{\prime 6}$

Adicionalmente a lo anteriormente expuesto, la Secretaría debe comprender que los "Términos y Condiciones" establecidos en las páginas web o bien en los mismos aplicativos de las empresas fungen de contratos entre las personas que descargan el programa y aceptan las cláusulas, convirtiéndose así en usuarios conductores y usuarios pasajeros. Por consiguiente, quienes usan el aplicativo móvil son informados previa e inequívocamente antes de la prestación del servicio, conociendo de antemano los derechos y obligaciones que enmarcan esa relación contractual.

Es por esta razón que mediante las Resoluciones No 099-2015/CD1-INDECOPI, 074-2015/ CD1-INDECOPI, 075-2015/CD1-INDECOPI y 100-2015-CD1-INDECOPI, la Comisión de Fiscalización de Competencia Desleal $N^{\circ} 1$ de INDECOPI rechazó los argumentos 1, 2 y 3 descritos anteriormente y señalados por la Secretaría en contra las empresas de aplicativo de movilidad $^{7}$ declarando infundadas las imputaciones establecidas de oficio por presunta comisión de actos de competencia desleal en las modalidades en engaño e infracción de normas supuestos ejemplificados en los artículos 8 y el literal b) del numeral 14.2 del artículo 14 del Decreto Legislativo 1044 —Ley de Represión de la Competencia Desleal一.

5. Enrique de la Puerta, «El diseño de modelo de negocio disruptivo», Business Review 289 (2019), https://www. harvard-deusto.com/modelos-de-negocio-disruptivos.

Thomson Reuters, "Cinco tecnologías disruptivas que cambiarán los modelos de negocio», Thomson Reuters, 2020, https://www.thomsonreuters.com.ar/es/soluciones-fiscales-contables gestion/blog-empresas/cinco-tecnologias-disruptivas-que-cambiaran-los-modelos-de-negocio.html.

6. Resolución N099-2015/CD1-INDECOPI, 13. 
El pronunciamiento unánime de INDECOPI recaído en cuatro resoluciones sucesivas en el año 2015 no hace sino más que reafirmar un entendimiento sobre la naturaleza de este tipo de industria, la cual corresponde a un modelo disruptivo de negocio ${ }^{8}$ que nace por medio de la tecnología y que viene satisfaciendo la demanda de millones de usuarios alrededor del mundo. Una posición disímil a la tomada por INDECOPI hubiera significado un aumento en los costos de transacción debido a la imposición de barreras de acceso al mercado y por supuesto un cambio crucial en el ADN de estas empresas, además de otros varios aplicativos operantes a nivel local los cuales tendrían que buscar forzadamente "encasillarse" en un tipo de industria ya existente - transporte, por ejemplo-, cuando se encuentran forjando una propia.

\section{DE LAS RELACIONES JURÍDICAS EXISTENTES}

Finalmente, es importante hacer referencia a que desde que se solicita un servicio por intermedio del aplicativo hasta que se llega al destino final, nos encontramos frente a tres relaciones jurídicas diferentes e independientes entre sí, las cuales se detallan a continuación:

\section{Relación jurídica 1: aplicativo - usuario conductor.}

Es una relación jurídica de naturaleza civil que se entabla en virtud a los términos y condiciones celebrados entre ambas partes. Esto es, el usuario conductor accede a una aplicación que le permite - a través de un teléfono celular o móvil- recibir solicitudes de servicio de transporte de los usuarios pasajeros afiliados a dicha aplicación, procediendo a realizar el servicio si acepta libremente la solicitud antes descrita, fungiendo la empresa de aplicativo móvil de intermediario.

\section{Relación jurídica 2: aplicativo - usuario pasajero.}

Es una relación jurídica de consumo que se entabla en virtud a los términos y condiciones celebradas entre ambas partes. En virtud a las disposiciones mencionadas en los términos y condiciones, el usuario pasajero accede a una aplicación que le permite, a través de un teléfono celular o móvil, enviar solicitudes de servicio de transporte de los usuarios conductores afiliados a dicha aplicación, a efectos que estos últimos las acepten y les brinden libremente el servicio, fungiendo la empresa de aplicativo móvil de intermediario.

\section{Relación jurídica 3: usuario conductor - usuario pasajero.}

Es una relación jurídica de consumo que se entabla entre ambas partes, completamente independiente a las relaciones jurídicas antes descritas. En virtud a esta relación jurídica, el usuario conductor presta el servicio efectivo de transporte terrestre al usuario pasajero a cambio de un precio determinado previamente indicado en el aplicativo.

Entonces, no existe una obligación legal ni reglamentaria que exija a las compañías del gremio de ride-hailing apps contar con títulos habilitantes por cuando su actividad principal y ordinaria no es brindar los servicios que realiza una "empresa de taxi". Además, los usuarios conductores no laboran para las empresas de aplicativo de movilidad. Ninguna empresa de aplicativo móvil contrata conductores ni usuarios conductores ni tiene relación laboral o de dependencia con ninguno de ellos. Por el contrario, ellos son usuarios de las aplicaciones al igual que los usuarios pasajeros, quienes tienen relaciones independientes entre sí y con las empresas de aplicativos.

\footnotetext{
7. Ibíd., 1.

8. Ibíd., 5.
} 


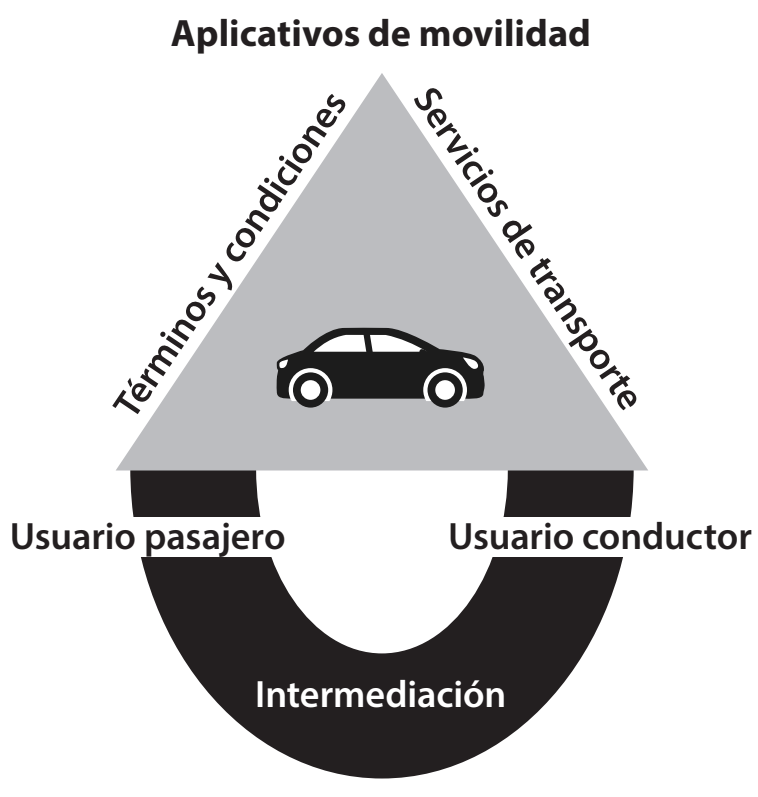

\section{CONCLUSIONES}

Los aplicativos de movilidad llegaron para quedarse, no solo en Perú, sino en el mundo. Vivimos una revolución tecnológica que no se detendrá; sino que por el contrario continuará desarrollando nuevas formas de intercambiar productos y servicios, motivo por el cual es imprescindible encontrar un entendimiento adecuado sobre la naturaleza de esta industria.

Como hemos observado en el análisis precedente, los modelos de negocio disruptivos se originan desde el año 2000 con el propósito de realizar una transformación digital sobre modelos tradicionales, por lo que no estamos refiriéndonos a un mismo tipo de sector. Mientras que las "empresas de taxi" dirigen las rutas y conductores, los aplicativos de movilidad intermedian eficientemente ese servicio, otorgando libertad a sus usuarios respecto al vehículo, conductor, ruta y clase de servicio a ofertar.

La adaptación y el replanteamiento por parte de las entidades de gobierno encargadas de su supervisión y los policy makers, serán palabras claves para el desarrollo de una adecuada convivencia de la industria y los usuarios de los aplicativos, que incrementan día a día. 